\title{
A retrospective study of electroencephalogram parameters during general anaesthesia in infants and young children
}

\section{Zhengzheng Gao \\ Beijing Children's Hospital \\ Beijing Children's Hospital \\ Xiaoxue Wang \\ Beijing Children's Hospital \\ Mengnan Yao \\ Beijing Children's Hospital \\ Lan Sun \\ Beijing Children's Hospital \\ Dongyu Qiu \\ Beijing Children's Hospital \\ Yi Ren \\ Beijing Children's Hospital}

Jianmin Zhang ( $\nabla$ sunbrightangel@sina.com )

\section{Research Article}

Keywords: PSI, SEF, burst suppression, general anaesthesia, children

Posted Date: January 6th, 2021

DOl: https://doi.org/10.21203/rs.3.rs-137166/v1

License: (1) This work is licensed under a Creative Commons Attribution 4.0 International License. Read Full License 


\section{Abstract \\ Background}

This retrospective study describes electroencephalogram (EEG) parameters in infants and young children under general anaesthesia. The study's primary objective was to detect determinants of patient state index (PSI) levels.

\section{Methods}

We analysed EEG parameters in patients aged 1-36 months who received sevoflurane or propofol as the primary anaesthetic in Beijing Children's Hospital from September 1, 2019, to April 30, 2020. Patients were stratified into two age groups: 1-12 and 13-36 months. The relationship between EEG parameters and clinical parameters was analysed by multivariable linear regression.

\section{Results}

Sixty-two patients were involved in the final analysis, including 35 boys and 27 girls. Average patient age and weight were $14.5(5.8-24.0)$ months and $9.9(8.0-12) \mathrm{kg}$, respectively. Spectral edge frequency (SEF), PSI and blood pressure were lower $(p<0.001)$ and burst suppression rate (BSR) and heart rate higher $(p<0.001)$ at the age of 1-12 months under general anaesthesia. The most significant factor associated with higher levels of PSI was a higher SEF at the age of $1-36$ months $(1-12$ months $p=0.001 ; 13-26$ months $p<0.001)$; burst suppression occurrence decreased the PSI level $(p=0.031)$. Older age or higher blood pressure increased PSI levels in children under 1 year old $(p=0.028$ and $p=0.002)$.

\section{Conclusions}

SEF was the most significant determinant associated with PSI levels in infants and young children during general anaesthesia, and burst suppression occurrence decreases the PSI level. Moreover, age and blood pressure might affect PSI levels in infants.

\section{Introduction}

In June 2016, the US FDA issued a warning to the paediatric medical community that anaesthesia and sedation in children younger than 3 years old can cause adverse neurodevelopmental outcomes. ${ }^{[1]}$ This warning has caught the attention of the world's paediatric anaesthesia community, which hopes to reduce the risk of neurotoxicity by changing anaesthesia methods. Overall, non-invasive and reliable monitoring of the depth of anaesthesia is still a clinical concern for paediatric anaesthesiologists. ${ }^{[2]}$ The effects of anaesthesia and electrical brain activity can be assessed using EEG monitors, which are designed to guide general anaesthesia. ${ }^{[3]}$ However, EEG-based indices, such as BIS and narcotrend, for monitoring the depth of anaesthesia assume that the same index value defines the same level of unconsciousness for all 
anaesthetics. A previous adult study has confirmed that the PSI might be a useful alternative to the bispectral index for assessing the level of consciousness during the induction of and emergence from anaesthesia, as well as for titrating IV and volatile anaesthetics during surgery. ${ }^{[4]}$ Although EEG-based indices have been in use for years, they do not ensure that awareness under general anaesthesia can be prevented; ${ }^{[5]}$ in addition, these indices, which have been developed using adult patient cohorts, do not relate directly to the neurophysiology of how a specific anaesthetic exerts its effects in the brain, and they thus cannot provide an accurate picture of the brain's responses to the drugs.

We sought to understand how well the PSI works in infants and young children. The objective of this study was to evaluate determinants of PSI under general anaesthesia. PSI, a quantitative electroencephalographic index, has been introduced into clinical practice as a monitor for assessing consciousness during sedation and general anaesthesia. ${ }^{[4]} \mathrm{SEF}$ is the frequency such that $95 \%$ of the power in the spectrum lies below this value. ${ }^{[6]} \mathrm{BSR}$ reflects the relative duration of electrocortical suppression between bursts of activity and measures how much the electrical activity of the frontal and pre-frontal cortex of the brain is suppressed as a percentage of time. ${ }^{[7]}$

\section{Materials And Methods}

\section{Patients}

This study was approved by the Ethics Committee of Beijing Children's Hospital, China (2020-Z-033) on May 9,2020 . We confirm that all methods were performed in accordance with relevant guidelines and regulations of Beijing Children's Hospital, and informed patient consent was waived due to the retrospective nature of the study. The study was registered with the Chinese Clinical Trial Registry under the number ChiCTR2000033427. We reviewed our database for patients who underwent general anaesthesia with simultaneous EEG recordings collected by a Sedline (Masimo Corporation, Irvine, CA) from September 1 , 2019, to April 30, 2020. The inclusion criteria were as follows: (1) aged 1-36 months, (2) general anaesthesia. The exclusion criteria were as follows: (1) ASA $\geq 3$; (2) cardiac, intracranial, and emergency procedures. Due to expected age-dependent changes in EEG under general anaesthesia, patient enrolment was stratified into two age groups: A, 1-12 months; and B, 13-36 months. ${ }^{[8,9]}$

\section{Data acquisition}

EEG data were downloaded with Masimo instrument configuration tool software (Masimo Corporation, Irvine, $\mathrm{CA})$ and manually reviewed. Demographic information and physiological data were collected from the electronic medical records system.

For each subject, we identified a post-induction period beginning $\sim 15 \mathrm{~min}$ after induction and concluding at the end of the procedure, which was based on the protocol of Blain-Moraes et al. ${ }^{[10]}$ We then selected a 10min period in this phase with steady haemodynamics and without muscle activity, and applied artificial interference to analyse the relationship among PSI, pooled SEF (mean value of the left and right side) and BSR under general anaesthesia. The EEG and physiological data represent average values. 


\section{Statistical analysis}

Histograms and the Kolmogorov-Smirnov test were used to assess normality. Continuous variables are expressed as the mean \pm standard deviation or median (interquartile interval), as appropriate. Differences between unpaired groups were analysed using a Mann-Whitney Uor Kruskal-Wallis test for continuous variables and a chi-square test for nominal variables. Linear regression analysis was applied in univariable and multivariable manners to identify determinants of PSI level. Variables with statistically significant values in the univariable model that did not show any multicollinearity were entered into the multivariable model. The multivariable model was based on forwarding stepwise regression. A $p$-value of less than 0.05 indicated a significant difference. The statistical analysis was performed using IBM SPSS Statistics 22.0 (SPSS Inc., Chicago, IL) and GraphPad Prism 8.0 (GraphPad Software Company, San Diego, CA).

\section{Results}

\section{Patient demographics and characteristics}

All 75 EEGs were reviewed for artefacts and noise. We excluded 2 patients who were improperly fitted with EEG electrodes resulting in poor data quality, 4 patients who underwent an emergency procedure, 2 patients who underwent a cardiac procedure, 3 patients who underwent an intracranial procedure, and 2 patients $A S A \geq 3$. Ultimately, 62 patients were deemed suitable for analysis (Figure 1).

The demographic and clinical characteristics of the patients are given in Table 1. The patient's age and weight were 14.5 (5.8-24.0) months and 9.9 (8.0-12) kg, respectively, and the study included 35 boys and 27 girls. All patients underwent general anaesthesia with sevoflurane or propofol for maintenance. Compared with group B, the patients in group A were younger and had a lower weight. Baseline data showed no statistically significant difference between the two groups in terms of sex, ASA, or maintenance drug.

Electroencephalogram parameters and physiological data are shown in Table 2. PSI, SEF were significantly lower $(p<0.001)$ and BSR higher in group A $(p<0.001)$. In contrast, heart rate was lower $(p<0.001)$ and blood pressure higher in group B (SBP $p<0.001$; DBP $p=0.01$; MAP $p<0.001)$.

\section{Association of PSI level with different variables}

To avoid multicollinearity, only MAP was used to represent blood pressure in the analysis. In group A, univariable linear regression analysis revealed that higher PSI levels correlated significantly with a higher SEF, older age, greater weight, and higher blood pressure (Table 3; SEF $p<0.001$; age $p<0.001$; weight $p<0.001$; MAP $p=0.009)$. However, a higher heart rate and BSR were associated with lower PSI levels (HR $p=0.036$; BSR $p<0.001$ ). In group B, according to linear regression analysis, a higher PSI level correlated significantly with a higher SEF and greater weight (SEF $p<0.001$; weight $p=0.032$ ).

In group $\mathrm{A}$, the multivariable model $\left(R^{2}=0.909\right)$ showed that the most significant factors associated with higher levels of PSI were a higher SEF, older age, and higher blood pressure (SEF $p=0.001$; age $p=0.028$, MAP $p=0.002)$, whereas a higher BSR was the most significant factor independently associated with a lower PSI level $(p=0.031)$. In group $B$, the multivariable model $\left(R^{2}=0.826\right)$ revealed that the most significant factor 
independently associated with higher levels of PSI was a higher SEF $(p<0.001)$. The residual plots for the two groups are shown in Figure 2.

\section{Discussion}

\section{EEG parameters}

In our study, significantly higher PSI levels were found in 13-36-month-old children in comparison to 1-12month-old children, and SEF was also higher in the former group during general anaesthesia. Relatively low PSI levels in infants may occur during general anaesthesia, which do not indicate deep anaesthesia. These changes in SEF might be explained by several structural and functional neurobiological factors associated with age. For example, the neurodevelopmental processes that occur throughout childhood, including thalamocortical development, may underlie the size of the EEG signal enhancement by age. ${ }^{[11]}$ Alpha (8-13 $\mathrm{Hz}$ ) coherence, a prominent EEG feature associated with sevoflurane-induced unconsciousness in adults, is absent in patients $<1$ year, ${ }^{[8]}$ and only slow $(<1 \mathrm{~Hz})$ and delta $(1-4 \mathrm{~Hz})$ oscillations can be observed; therefore, SEF in children $<1$ year is lower. Overall, SEF is lower in an undeveloped cerebral cortex under general anaesthesia, and the age-related changes observed in the SEF are consistent with the neurobiology and neurophysiology of growth. During general anaesthesia, a higher BSR was found at the age of 1-12 months. Both Cornelissen et al and Davidson et al reported a higher prevalence of isoelectric events at younger ages. $[12,13]$ We suggest that patients between 1 and 12 months of age with an immature brain are more vulnerable to the effects of anaesthetic.

\section{Determinants of PSI level}

\section{1-12-month-old group}

In linear regression analysis, increased PSI levels were significantly related to an older age, greater weight, lower heart rate, higher blood pressure, higher SEF level, and lower BSR level in the univariable model (Table 3). However, multivariable analysis revealed that a higher SEF was the most significant determinant associated with a higher PSI level. In addition, a higher BSR was associated with a lower PSI level. Age and blood pressure also exhibited a positive correlation with PSI in infants during general anaesthesia. In summary, in children under 1 year old, the SEF mainly determines the PSI level, and burst suppression might lower PSI; older age and higher blood pressure might elevate PSI during general anaesthesia. Our results are consistent with a previous study illustrating burst suppression can drive the index value lower. ${ }^{[14]}$

\section{3-36-month-old group}

In linear regression analysis, increased PSI levels were significantly related to a greater weight and higher SEF level in the univariable model (Table 3). Regardless, based on multivariable analysis, a higher SEF was the most significant determinant independently associated with a higher PSI level. In children 13 to 36 months, the SEF mainly determines the PSI level during general anaesthesia.

According to the above results, age and blood pressure have a strong effect on PSI level during general anaesthesia in children aged 1-12 months. We, therefore, speculate that age-dependent changes in SEF are 
more obvious in infants than in young children, which leads to changes in PSI; thus, age is a determent associated with PSI in infants. These age-dependent changes likely relate to changes in brain structure and function that occur during development, which is consistent with some previous studies; synaptogenesis begins approximately post birth, and synaptic density increases to maximum levels by approximately 2 years of age in humans. ${ }^{[15-17]}$ In addition, cerebral perfusion pressure (CPP) is likely more dependent on blood pressure in infants under general anaesthesia, whereby a higher CPP might increase the cerebral blood flow, resulting in a higher PSI level; hence, blood pressure is a determinant associated with PSI in infants. In contrast, PSI has no relationship with age and blood pressure under general anaesthesia in young children.

\section{Study limitations}

Our study had some limitations that need to be addressed. First, we did not analyse patients undergoing craniocerebral, cardiac, or emergency surgery or patients with poor preoperative conditions. Second, we solely observed EEG parameters under the action of propofol or sevoflurane. Moreover, we only focused on the maintenance phase of anaesthesia. Data for new-born and older children will be analysed in our future studies.

\section{Conclusion}

SEF was the most significant determinant independently associated with PSI level in infants and young children during general anaesthesia. More burst suppression occurred in infants, which might decrease the PSI level. In addition, age and blood pressure might affect PSI in children under 1 year old.

\section{Declarations}

Ethical approval and consent to participate: This study was approved by the Ethics Committee of Beijing Children's Hospital, China (No.2020-Z-033). Informed patient consent was waived due to the retrospective nature of the study.

Consent for publication: Not applicable, as there are no details, images, or videos of individuals within the manuscript.

Availability of data and materials: The datasets supporting the conclusions of this article are included within the article.

Competing interests: The authors certify that there is no conflict of interest with any financial organisation regarding the material discussed in the manuscript.

Funding: Not applicable.

\section{Author contributions:}

Gao Zhengzheng and Zhang Jianmin wrote the main manuscript text. Wang Xiaoxue, and Yao Mengnan analyzed the data. Sun Lan, Ren Yi, and Qiu Dongyu prepared figures and tables. All authors read and approved the final manuscript. 
Acknowledgments: We would like to thank the reviewers for their careful review and critiques, which helped us improve the manuscript considerably.

\section{References}

[1] FDA Drug Safety Communication: FDA approves label changes for use of general anesthetic and sedation drugs in young children. https://www.fda.gov/Drugs/DrugSafety/ucm554634.htm.

[2] Gu Y, Liang Z, Hagihira S. Use of Multiple EEG Features and Artificial Neural Network to Monitor the Depth of Anesthesia. Sensors (Basel). 2019. 19(11).

[3] Purdon PL, Sampson A, Pavone KJ, Brown EN. Clinical Electroencephalography for Anesthesiologists: Part I: Background and Basic Signatures. Anesthesiology. 2015. 123(4): 937-60.

[4] Chen X, Tang J, White PF, et al. A comparison of patient state index and bispectral index values during the perioperative period. Anesth Analg. 2002;95:1669-1674, table of contents.

[5] Avidan MS, Jacobsohn E, Glick D, et al. Prevention of intraoperative awareness in a high-risk surgical population. N Engl J Med. 2011. 365(7): 591-600.

[6] Matsuura T, Oda Y, Ikeshita K, et al. Differential electroencephalographic response to tracheal intubation between young and elderly during isoflurane and sevoflurane nitrous oxide anaesthesia. Br J Anaesth. 2007;99:858-863.

[7] Besch G, Liu N, Samain E, et al. Occurrence of and risk factors for electroencephalogram burst suppression during propofol-remifentanil anaesthesia. Br J Anaesth. 2011;107:749-756.

[8] Akeju O, Pavone KJ, Thum JA, et al. Age-dependency of sevoflurane-induced electroencephalogram dynamics in children. Br J Anaesth. 115 Suppl 1England,2015. i66-i76.

[9] Cornelissen L, Kim SE, Lee JM, Brown EN, Purdon PL, Berde CB. Electroencephalographic markers of brain development during sevoflurane anaesthesia in children up to 3 years old. Br J Anaesth. 2018. 120(6): 12741286.

[10] Blain-Moraes S, Tarnal V, Vanini G, et al. Neurophysiological correlates of sevoflurane-induced unconsciousness. Anesthesiology. 2015. 122(2): 307-16.

[11] Lee JM, Akeju O, Terzakis K, et al. A Prospective Study of Age-dependent Changes in Propofol-induced Electroencephalogram Oscillations in Children. Anesthesiology. 2017. 127(2): 293-306.

[12] Davidson AJ, Sale SM, Wong C, et al. The electroencephalograph during anesthesia and emergence in infants and children. Paediatr Anaesth. 2008. 18(1): 60-70.

[13] Cornelissen L, Bergin AM, Lobo K, Donado C, Soul JS, Berde CB. Electroencephalographic discontinuity during sevoflurane anesthesia in infants and children. Paediatr Anaesth. 2017. 27(3): 251-262. 
[14] Bruhn J, Bouillon TW, Shafer SL. Bispectral index (BIS) and burst suppression: revealing a part of the BIS algorithm. J Clin Monit Comput. 2000. 16(8): 593-6.

[15] Herschkowitz N, Kagan J, Zilles K. Neurobiological bases of behavioral development in the first year. Neuropediatrics. 1997. 28(6): 296-306.

[16] Glantz LA, Gilmore JH, Hamer RM, Lieberman JA, Jarskog LF. Synaptophysin and postsynaptic density protein 95 in the human prefrontal cortex from mid-gestation into early adulthood. Neuroscience. 2007. 149(3): 582-91.

[17] Petanjek Z, Judaš M, Šimic G, et al. Extraordinary neoteny of synaptic spines in the human prefrontal cortex. Proc Natl Acad Sci U S A. 2011. 108(32): 13281-6.

\section{Tables}

\begin{tabular}{|lllll|}
\hline \multicolumn{5}{|c|}{ Table 1. Patient demographics and anaesthetic factors in all patients } \\
& All Patients & Group A & Group B & p Value \\
\hline Age (m) & $14.5(5.8-24.0)$ & $5.5(3.0-9.1)$ & $23.5(18.0-30.5)$ & $<0.001^{*}$ \\
\hline Weight (kg) & $9.9(8.0-12)$ & $8.0(5.3-9.0)$ & $12.0(11.0-15.1)$ & $<0.001^{*}$ \\
\hline Male & $35(57 \%)$ & $16(53 \%)$ & $19(89 \%)$ & 0.63 \\
\hline Female & $27(43 \%)$ & $14(47 \%)$ & $13(41 \%)$ & \\
\hline ASA区 & $33(53 \%)$ & $16(53 \%)$ & $17(53 \%)$ & 0.99 \\
\hline ASAX & $29(47 \%)$ & $14(47 \%)$ & $15(47 \%)$ & \\
\hline Maintenance drug & & & & \\
\hline Sevoflurane & $28(45 \%)$ & $13(43 \%)$ & $15(47 \%)$ & 0.78 \\
\hline Propofol & $34(55 \%)$ & $17(57 \%)$ & $17(53 \%)$ & \\
\hline Abbreviations: ASA, American Society of Anesthesiologists & \\
\hline
\end{tabular}




\begin{tabular}{|c|c|c|c|}
\hline & Group A & Group B & $p$ Value* \\
\hline PSI & 29.5 (10.7) & $43.6(7.6)$ & $<0.001$ \\
\hline SEF & $11.4(4.6)$ & $17.4(3.0)$ & $<0.001$ \\
\hline BSR & $5.2(0.0-13.3)$ & $0.0(0.0-0.0)$ & $<0.001$ \\
\hline$H R, b \cdot \min ^{-1}$ & $128.0(17.6)$ & $105.0(15.9)$ & $<0.001$ \\
\hline SBP, mmHg & 74.5 (11.0) & $86.1(9.5)$ & $<0.001$ \\
\hline $\mathrm{DBP}, \mathrm{mmHg}$ & $41.6(11.8)$ & $49.7(12.1)$ & 0.01 \\
\hline MAP, mmHg & $52.5(7.3)$ & $63.4(9.4)$ & $<0.001$ \\
\hline \multicolumn{4}{|c|}{$\begin{array}{l}\text { Abbreviations: PSI, patient state index; SEF, 95\% spectral edge frequency; BSR, burst suppression rate; HR, } \\
\text { heart rate; SBP, systolic blood pressure; DBP, diastolic blood pressure; MAP, mean arterial pressure. }\end{array}$} \\
\hline \multicolumn{4}{|l|}{${ }^{*} p<0.05$} \\
\hline
\end{tabular}


Table 3. Univariable and multivariable linear regression analyses for determinants of PSI levels in two groups

\begin{tabular}{|c|c|c|c|c|c|c|c|c|}
\hline & \multicolumn{4}{|l|}{ Group A } & \multicolumn{4}{|l|}{ Group B } \\
\hline & \multicolumn{2}{|c|}{ Univariable analysis } & \multicolumn{2}{|c|}{$\begin{array}{l}\text { Multivariable } \\
\text { analysis }\end{array}$} & \multicolumn{2}{|c|}{ Univariable analysis } & \multicolumn{2}{|c|}{$\begin{array}{l}\text { Multivariable } \\
\text { analysis }\end{array}$} \\
\hline & $\begin{array}{l}\text { regression } \\
\text { coefficient }\end{array}$ & $\begin{array}{l}p \\
\text { value }\end{array}$ & $\begin{array}{l}\text { regression } \\
\text { coefficient } \\
(95 \% \mathrm{Cl})\end{array}$ & $\begin{array}{l}p \\
\text { value }\end{array}$ & $\begin{array}{l}\text { regression } \\
\text { coefficient }\end{array}$ & $\begin{array}{l}p \\
\text { value }\end{array}$ & $\begin{array}{l}\text { regression } \\
\text { coefficient } \\
(95 \% \mathrm{Cl})\end{array}$ & $\begin{array}{l}p \\
\text { value }\end{array}$ \\
\hline Age & 0.807 & $\begin{array}{l}< \\
0.001^{*}\end{array}$ & $\begin{array}{l}0.908 \\
(0.108- \\
1.708)\end{array}$ & $0.028^{*}$ & 0.308 & 0.086 & - & - \\
\hline Sex & -0.184 & 0.330 & - & - & 0.033 & 0.86 & - & - \\
\hline Weight & 0.686 & $\begin{array}{l}< \\
0.001^{*}\end{array}$ & $\begin{array}{l}0.191 \\
(-0.874- \\
1.256)\end{array}$ & 0.714 & 0.381 & $0.032^{*}$ & $\begin{array}{l}-0.049 \\
(-0.535- \\
0.438)\end{array}$ & 0.840 \\
\hline ASA & 0.054 & 0.775 & - & - & -0.118 & 0.522 & - & - \\
\hline Anesthetics & 0.169 & 0.373 & - & - & 0.235 & 0.196 & - & - \\
\hline $\mathrm{HR}$ & -0.384 & $0.036^{*}$ & $\begin{array}{l}-0.079 \\
(-0.165- \\
0.007)\end{array}$ & 0.071 & -0.164 & 0.369 & - & - \\
\hline MAP & 0.467 & $0.009^{*}$ & $\begin{array}{l}0.361 \\
(0.147- \\
0.575)\end{array}$ & $0.002^{*}$ & 0.206 & 0.258 & - & - \\
\hline SEF & 0.873 & $\begin{array}{l}< \\
0.001^{*}\end{array}$ & $\begin{array}{l}0.943 \\
(0.408- \\
1.478)\end{array}$ & $0.001^{*}$ & 0.909 & $\begin{array}{l}< \\
0.001^{*}\end{array}$ & $\begin{array}{l}2.286 \\
(1.848- \\
2.724)\end{array}$ & $\begin{array}{l}< \\
0.001^{*}\end{array}$ \\
\hline BSR & -0.692 & $\begin{array}{l}< \\
0.001^{*}\end{array}$ & $\begin{array}{l}-0.223 \\
(-0.423- \\
-0.023)\end{array}$ & $0.031^{*}$ & -0.148 & 0.419 & - & - \\
\hline \multicolumn{9}{|c|}{$\begin{array}{l}\text { Abbreviations: ASA, American Society of Anesthesiologists; HR, heart rate; SBP, systolic blood pressure; } \\
\text { DBP, diastolic blood pressure; MAP, mean arterial pressure; SEF } 95,95 \% \text { spectral edge frequency; BSR, } \\
\text { burst suppression rate. }\end{array}$} \\
\hline${ }^{*} p<0.05$ & & & & & & & & \\
\hline
\end{tabular}

Figures 


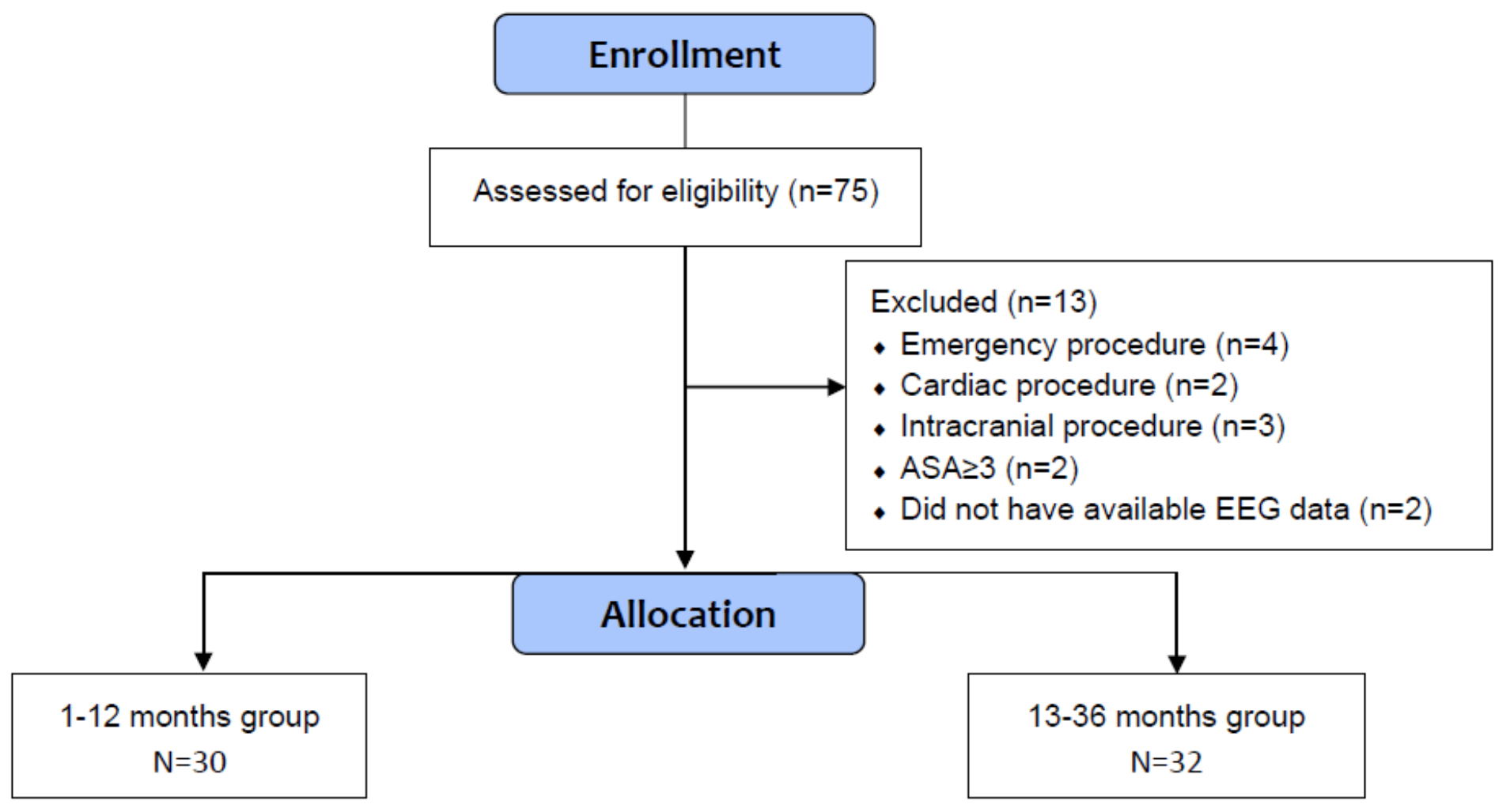

Figure 1

Flow diagram of the cases in the study
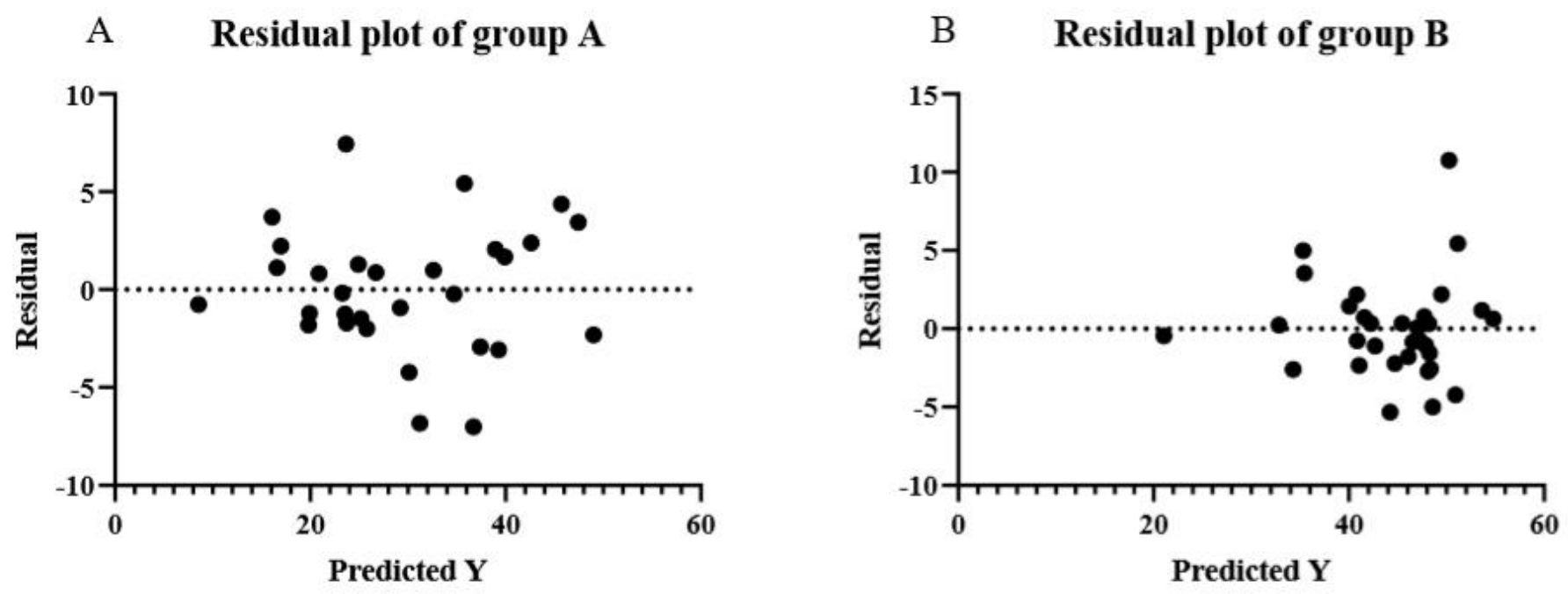

Figure 2

A. Residual plot of the 1-2-month-old group. B. Residual plot of the 13-36-month-old group. 